\title{
What Can Be Learnt from Experienced Data Scientists? A Case Study
}

\section{Riungu-Kalliosaari, Leah}

Springer

2017

Riungu-Kalliosaari , L , Kauppinen , M \& Männistö , T M 2017 , What Can Be Learnt from Experienced Data Scientists? A Case Study . in M Felderer, D Méndez Fernández , B Turhan, M Kalinowski , F Sarro \& D Winkler (eds), Product-Focused Software Process Improvement : 18th International Conference, PROFES 2017 Innsbruck, Austria, November pÿ29 December 1, 2017 Proceedings . Lecture Notes in Computer Science , no. 10611, Springer, Cham , pp. 55-70, International Conference on on Product-Focused Software Process Improvement , Innsbruck , Austria , 29/11/2017 . https://doi.org/10.1007/978-3-319-69926-4_5

http://hdl.handle.net/10138/235296

https://doi.org/10.1007/978-3-319-69926-4_5

acceptedVersion

Downloaded from Helda, University of Helsinki institutional repository.

This is an electronic reprint of the original article.

This reprint may differ from the original in pagination and typographic detail.

Please cite the original version. 


\title{
What can be Learnt from Experienced Data Scientists? A Case Study
}

\author{
Leah Riungu-Kalliosaari $^{1}$, Marjo Kauppinen ${ }^{2}$, and Tomi Männistö ${ }^{1}$ \\ 1 University of Helsinki, Helsinki, Finland, firstname.lastname@helsinki.fi \\ 2 Aalto University, Espoo, Finland, firstname.lastname@aalto.fi
}

\begin{abstract}
Data science has the potential to create value and deep customer insight for service and software engineering. Companies are increasingly applying data science to support their service and software development practices. The goal of our research was to investigate how data science can be applied in software development organisations. We conducted a qualitative case study with an industrial partner. We collected data through focus group interviews, feedback sessions and workshops. This paper presents the data science process recommended by experienced data scientists and describes the key characteristics of the process, i.e., agility, continuous learning process and end-to-end process. We also report the challenges experienced while applying the data science process in service and software engineering projects. For example, the data scientists highlighted that it is challenging to identify the right problem and ensure that the results will be utilised. The results indicate that it is possible to put in place an agile, iterative data science process that supports continuous learning while focusing on a real business problem to be solved. However, the application of data science can be demanding and requires skills for addressing human and organisational issues.
\end{abstract}

Keywords: data science, software development, service engineering

\section{Introduction}

Data science is defined as "a new interdisciplinary field that synthesises and builds on statistics, informatics, computing, communication, management and sociology to study data and its environments (including domains and other contextual aspects, such as organizational and social aspects) in order to transform data to insights and decisions by following a data-to-knowledge-to-wisdom thinking and methodology" [4]. The interdisciplinary nature implies that knowledge from different fields is needed in order to ensure successful outcomes, making data scientists valued members of teams in many different fields. In particular, there is a growth in the application of data science in software engineering [3]. For example, in 2015, Microsoft grew its 'data and applied science' discipline 
to over six hundred people and more than 1600 people were interested in data science work and signed up to data science related mailing lists [10].

Five years ago, Davenport and Patil [7] described the data scientist position as the sexiest job of the 21st century. In the recent past, the data scientist role has grown in both popularity and demand. However, there is a wide shortage of data scientists despite an increasing need for them across many fields [7]. In order to fill the growing gap, education institutions are also making efforts in educating future data scientists [14].

In order for data scientists to add the most value, they must be part of a team that encourages them to 'innovate with customer-facing products and services and not just to create reports and presentations' [7]. As part of a large Finnish research programme Need for $\mathrm{Speed}^{3}$, we wanted to understand how data science can enable organizations to gain deep customer insight. We conducted a case study with one of the project partners whose data science team was involved in service and software development projects. We wanted to understand the activities involved in the data science projects along with the challenges associated with them. Hence, we focused on these research questions: (1) What are the key characteristics of the data science process applied in service and software development projects? and (2) What are the challenges of applying the data science process in the projects?

We present the results of the study in this paper. We found the data science process to be an agile, end-to-end and continuous learning process. We classified the challenges into three groups: (1) the demanding problems, e.g., difficulties in identifying relevant problems and measuring the impact of the results; (2) moderate problems e.g. unrealistic customer expectations; (3) mild problems such as poor data quality and differences in modelling and production technologies.

The rest of the paper is as follows: Section 2 takes a look at related research; Section 3 presents the research process; Section 4 presents the results as lessons learnt; Section 5 discusses the results and Section 6 concludes the paper.

\section{Related Work}

As data science continues to gain more prevalence in software engineering, so does the role of data scientists within software teams. The data scientist role, description and term have evolved over time. Kandel et al. [9] referred to data scientists as analysts falling into three types: Hackers - who are proficient programmers and are comfortable manipulating data; Scipters - proficient in modelling and producing visualizations with software packages such as $\mathrm{R}$ or Matlab; and $A p$ plication user - work with smaller data sets using applications, such as SAS and SPSS. More recently, Kim et al. [10] identified five emerging roles of data scientists in software engineering, namely "(1) Insight Providers, who work with engineers to collect the data needed to inform decisions that managers make; (2) Modelling Specialists, who use their machine learning expertise to build predictive models; (3) Platform Builders, who create data platforms, balancing both

\footnotetext{
${ }^{3}$ http://n4s.fi
} 
engineering and data analysis concerns; (4) Polymaths, who do all data science activities themselves; and (5) Team Leaders, who run teams of data scientists and spread best practices". These roles demonstrate that data science requires skills that should be spread among different individuals in a team, other than by expecting one person to possess them all-hence busting the myth of the unicorn data scientist.

Data science has the potential to improve software engineering in many ways. Begel and Zimmermann [1] surveyed the areas in which software engineers desired input from data scientists. They found 12 potential areas where data science could be applied namely, bug measurements, development practices, development best practices, testing practices, evaluating quality, services related to cloud computing and continuous delivery, customers and requirements, software development lifecycle, software development process, productivity, teams and collaboration, and reuse and shared components. The most important concern for software engineers had to do with customers' use of their applications.

Handling of data and producing results involves different activities. These may include tasks such as discovering the data for analysis, wrangling or manipulating the data into an appropriate format, profiling data to ensure its quality and suitability for analysis, modelling the data, and reporting the results of the analysis [9]. Similarly, according to Fisher et al. [8], the analysis process may include five activities, i.e., acquiring data, choosing an architecture, shaping the data into the architecture, writing an editing code, and reflecting and iterating on the results. All these activities have challenges that can sometimes make data analysis an exhausting process.

Some of the existing challenges include data access restrictions, data quality issues, i.e., missing, incorrect or inconsistent data values, difficulties with identifying data sources and integrating data from multiple sources, problems with inferring the most important data while creating models and visualizations, and communication issues, e.g., while presenting the results [9, 8].

The presence of data everywhere has led to a rapid growth of the data science field. Data-driven decision making is becoming increasingly critical while addressing different information needs in the software domain[3]. Critical and careful analysis of the problems should be practised in order to effectively apply

data science interventions. As the goal in such interventions is not primarily to analyse data, but make the data useful for decision-making in relation to the business processes, it is of importance to consider the problems from a wider perspective than, e.g., data analytics only. Hence, our focus is on the data science process, i.e., the activities and tasks carried out while analysing data to produce actionable insights and outcomes.

\section{$3 \quad$ Research Process}

We conducted a qualitative study with experienced data scientists to understand their data science process along with its challenges (see Table 1 for an overview of our research process). We use the term 'experienced data scientist' because 
the participants had each been involved in data science or analytics type of work for 4-12 years (see Table 2). The data scientists were employees of an industrial partner Reaktor ${ }^{4}$ in the Need for Speed programme. The industrial partner has 400 employees spread out in 4 offices across 3 continents. The company provides consultancy services in different areas with a connection to digital products and services. The data science team was composed of seven people.

At the beginning of the Need for Speed programme, the industrial partner hosted a workshop where its data science practices were presented and discussed (Phase I, Table 1). After the workshop, collaboration between the researchers and the company was agreed upon. In addition, the presentation material was made available to the researchers to be used as background and to triangulate the data from the other data collection phases.

Next, we carried out a focus group interview (Phase II, Table 1). We chose the focus group method because it is suitable for gathering experiences and discovering new insights as well as allowing an in-depth discussion within a reasonable period of time $[11,12]$. The goal of the focus group was to know more about the data science practice in the organization. The themes of the focus group included individual introductions, the company, the data science team, skills of a good data scientist, example projects, and lessons learnt (including challenges and success factors). Four researchers and four data scientists were present during the focus group interview. One researcher acted as the moderator and the others took notes and asked clarifying questions. The focus group was audio recorded and later transcribed for analysis. Details of the data scientists and the projects they had worked or were working on are shown in Table 2.

Table 1. Research process

\begin{tabular}{|c|l|l|l|l|l|}
\hline Phase & Theme & Method & Data & Informants & Outcomes \\
\hline I & $\begin{array}{l}\text { Background } \\
\text { overview of data } \\
\text { science practices }\end{array}$ & $\begin{array}{l}\text { Workshop, } \\
\text { presentations, } \\
\text { discussions }\end{array}$ & 5 slidesets & $\begin{array}{l}\text { Data science prac- } \\
\text { tices }\end{array}$ \\
\hline II & $\begin{array}{l}\text { Data science pro- } \\
\text { cess, challenges, } \\
\text { success factors }\end{array}$ & $\begin{array}{l}\text { Focus group } \\
\text { interview }\end{array}$ & $\begin{array}{l}\text { Audio } \\
\text { recording, } \\
\text { post-it } \\
\text { pictures }\end{array}$ & $\begin{array}{l}\text { DS1, DS2, } \\
\text { DS3, DS4 }\end{array}$ & $\begin{array}{l}\text { Process, its charac- } \\
\text { teristics, challenges, } \\
\text { success factors, } \\
\text { skills, projects }\end{array}$ \\
\hline III & $\begin{array}{l}\text { Validation of an- } \\
\text { alytic interpreta- } \\
\text { tions (for Phase } \\
\text { II), current situ- } \\
\text { ation }\end{array}$ & $\begin{array}{l}\text { Prosentation, } \\
\text { interview }\end{array}$ & $\begin{array}{l}\text { Slides, audio } \\
\text { recording }\end{array}$ & $\begin{array}{l}\text { DS1, DS4, } \\
\text { Research } \\
\text { manager }\end{array}$ & $\begin{array}{l}\text { Feedback on anal- } \\
\text { ysis, discussion on } \\
\text { current situation }\end{array}$ \\
\hline
\end{tabular}

\footnotetext{
${ }^{4}$ http://reaktor.com
} 
Table 2. Details of focus group participants

\begin{tabular}{|c|l|c|l|}
\hline Participant & Background & $\begin{array}{l}\text { Experience } \\
\text { (years) }\end{array}$ & $\begin{array}{l}\text { Goals of the case participant's } \\
\text { product development project }\end{array}$ \\
\hline DS1 & $\begin{array}{l}\text { Theoretical physics, } \\
\text { data mining }\end{array}$ & 12 & $\begin{array}{l}\text { Personalisation, optimisation; make } \\
\text { predictions }\end{array}$ \\
\hline DS2 & $\begin{array}{l}\text { Machine learning, } \\
\text { CS, statistics }\end{array}$ & 4 & $\begin{array}{l}\text { Change detection, make recommen- } \\
\text { dations, produce more tailored ad- } \\
\text { vertisements }\end{array}$ \\
\hline DS3 & $\begin{array}{l}\text { Machine learning, } \\
\text { statistics }\end{array}$ & 8 & $\begin{array}{l}\text { Marketing campaigns, make recom- } \\
\text { mendations, location analysis }\end{array}$ \\
\hline DS4 & $\begin{array}{l}\text { Psychology, IS, ma- } \\
\text { chine learning }\end{array}$ & 11 & $\begin{array}{l}\text { Segmentation; make recommenda- } \\
\text { tions, improve revenue and user ex- } \\
\text { perience }\end{array}$ \\
\hline
\end{tabular}

The data scientists were given post-it notes where they wrote notes related to the discussed themes. The post-it notes were collected, placed on a white board and a picture was taken that would be used to support the analysis.

After the analysis, we held a two-hour feedback workshop session (Phase III, Table 1). The session was to designed to act as a member checking type of a validation strategy [5] in the research process. The goal was to present the results of the analysis from the focus group session and get feedback from the data scientists. Three researchers, two data scientists (DS1 and DS4), and the company's research manager were present during the feedback session. The feedback session was also audio recorded and transcribed for analysis.

We analysed the data iteratively using the thematic analysis approach [6]. To guide our analysis, we used the pre-existing themes of interest discussed in the focus group interview, i.e., key characteristics of the data science process, challenges, success factors, example projects, and skills of a good data scientist. We iterated and refined the codes as we discussed with each other during the analysis as well as after the feedback session. We also used material obtained from the company to supplement our analysis, e.g., presentation slides. In this paper, we present the analysed themes related to the data science process, its characteristics and challenges.

\section{Lessons Learnt}

\subsection{Data Science Process}

The organisation had defined a data science process. During a Need for Speed programme workshop, the organisation presented the data science process on a high abstraction level. During the focus group and feedback sessions, the study 
participants provided more details about the process composed of six steps (Figure 1): conceptualization, problem definition, data collection and preparation, modelling, evaluation and validation, and deployment and utilization of results.

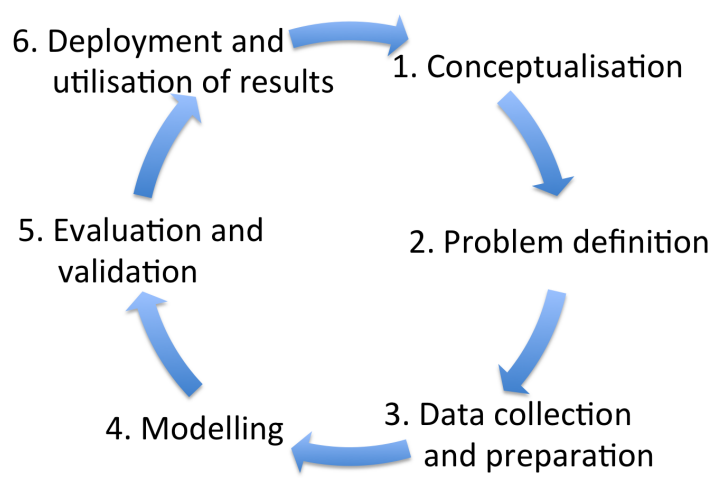

Fig. 1. Overall data science process of the case company.

Conceptualization: The main focus of this stage is the business problem. This involves interacting with the customer in order to assess the customer's understanding of (1) the business problem and (2) data science as a solution to the business problem. The business problem should be described clearly, putting the business targets and constraints into consideration, so as to develop the appropriate solution. The data scientists stressed the importance of knowing the customer's understanding of data science because it helped in preparing to address different customer expectations. One participant emphasised this:

It's important [for the customer] to understand the possibilities and limitations, really understanding what you are able to do and not do with data science. [DS3]

Problem definition: This stage focuses on the data science solution to the identified business problem. The business problem is formalised into an analytically solvable problem. One data scientists explained that many customers needed help to 'translate the [business] problem into a computational or mathematical problem' [DS1]. Successful problem definition therefore calls for a lot of interaction between the customer and the data scientist.

A good data science solution starts by understanding who the customer or end-user is. This helps to know how the data science solution will be applied. With this knowledge, the data scientists said that it was the best way to provide an optimum solution.

Data collection and preparation: The end result is determined by the data at hand. Hence, this makes collecting the data and preparing it for efficient use a vital aspect. In order to make this a fruitful endeavour, the data scientists 
wished that not only would the data be handed over to them, but that they would also be granted access to the actual data collection process. This would grant them the opportunity to improve the data collection process which they believed would have significant impact on the results.

Modelling: When the data is in good shape for analysis, the data scientists then manipulate the data using different data analysis and modelling techniques. Depending on the problem, modelling aims at describing what has happened, diagnosing why something has happened, predicting what will happen or providing guidance on how to make something happen. Often, the models are demonstrated using visualisations.

Evaluation and validation: The data scientists need to provide results that are reliable and relevant to the business problem. The participants were very interested in knowing the effectiveness of their results and therefore desired to obtain feedback from the real end users, not just from the business stakeholders or domain experts.

Deployment and utilization of results: It is essential that the results are put into use so as to assess their impact. Continuous and consistent monitoring is imperative along with a feedback loop that enables the end users to communicate their thoughts about the results. One participant [DS1] emphasised that tight collaboration with the end result user was very important.

\subsection{Characteristics of Data Science Process}

Agility: Data science projects are exploratory in nature. Following an agile approach helps to manage goals and expectations while addressing changes in a fruitful manner. The participants said the data science way of working resonated well with the agile approach.

There's a lot in common that you can really apply...Like always, do the MVP ["Minimum Viable Product"]...start iterating quick and try to have lots of communication, have the end user involved. [DS4]

... agility fits very well /with our] approach because we have to start with something and then actually try to produce as quickly as possible some kind of insight or results and then learn from those results and build on top of that. [We also] learn the environment that the customer has. Then actually I think it's more visible also to the customer [that] we are producing something useful. [DS3]

Data science problems have to deal with a degree of uncertainty. The agile approach provides the opportunity to address the unexpected changes along the way.

Continuous learning process: The agile approach mentioned above supports continuous learning throughout a project. It is most beneficial that both the data science team and the customer have the opportunity to learn something during the process, be it about the product, system, solution, process or people. It should be everyone's aim to 'learn by doing' [DS1] and use the new knowledge to improve the end results and possibly 'inspire some other ideas'. [DS1]

End to end process: This means that the data scientists start the project by first understanding the customer and the customer's problem. This entails 
evaluating the importance and relevance of the business problem. It calls for understanding the problem from the end-user's point of view in order to provide the appropriate solution.

...we have sort of tried to formulate our way of getting into projects that go on and we really want to put an emphasis on the starting point or the end usage point, of who is going to use this result and how. And we start from there and then go backwards and do what we can and then try to improve it always...really start from the end user. [DS1]

\subsection{Challenges}

We present the challenges as they were experienced by the data scientists in different phases of the data science process. Table 3 shows an overview of the challenges.

Table 3. Overview of the challenges

\begin{tabular}{|l|l|}
\hline Data Science Process Phase & Challenges \\
\hline Conceptualization & $\begin{array}{l}\text { Unrealistic customer expectations, communicat- } \\
\text { ing uncertainty }\end{array}$ \\
\hline Problem definition & $\begin{array}{l}\text { Identifying the right problem, limited interaction } \\
\text { with domain experts, preference for tools as a so- } \\
\text { lution }\end{array}$ \\
\hline Data collection and preparation & $\begin{array}{l}\text { Limited access to the data collection process, poor } \\
\text { data quality, lack of cooperation from all required } \\
\text { parties }\end{array}$ \\
\hline Modelling & $\begin{array}{l}\text { Lack of the required computational resources, dif- } \\
\text { ferences in modelling and production technologies }\end{array}$ \\
\hline Evaluation and validation & Lack of feedback from the end user \\
\hline $\begin{array}{l}\text { Deployment and utilization of re- } \\
\text { sults }\end{array}$ & $\begin{array}{l}\text { The results are not utilised, what is the impact of } \\
\text { the results? }\end{array}$ \\
\hline
\end{tabular}

Conceptualization The challenges at this stage had to do with unrealistic customer expectations and communicating uncertainty.

Unrealistic customer expectations: The participants found that most customers did not have a realistic view of data science and its capabilities. In order to sell their solutions, tool vendors had propagated a tools-driven approach in the market. Hence, the customers expected quick solutions, mostly in the form of tools or systems but not recommendations or guidelines to aid in decision 
making. This led to a tendency to acquire tools without clearly knowing the initial problem for which to use the tools.

...people have need for data science but they don't understand it...then the other thing is that the market is kind of saturated by vendors who don't really sell data science in the sense that we understand it. [DS4]

If customers did not understand data science well, it made it difficult for them to view the problem correctly, hence hindering how well they could conceptualise the problem. The participants strongly advocated for a data-driven approach and had to employ some effort in getting the customer to gain the appropriate focus on the problem.

Communicating uncertainty: Due to the exploratory nature of data science, it is not always easy to predict the results. The conceptualization process also involved getting the customer to have an open mind towards what the results might imply. It was difficult for the participants to get the customer to understand and accept the inherent uncertainty of the outcome. This resulted in prolonged initial negotiations that were not always fruitful in closing the deals.

...often times, it is that you [i.e., the data scientist] really cannot say beforehand that-okay this is the result and that is what you will get. Basically because the outcome is very vague. You [i.e., the customer] use the money and you don't know what you are investing [in]. [DS1]

Problem Definition The main challenge here was identification of the right problem to be addressed. The other issues were the limited interaction with the domain experts and the customers' overemphasis on tools.

Identifying the right problem: A correct problem should be one that is solved by the obtained results. The participants had a great desire to produce useful results. However, it was often that the customers could not clearly explicate the problem in the first place.

...in many cases, you notice that your customer has collected data, but what to do with the data is unclear. And then there are lots of things we can actually calculate from the data but, all of them are not useful ones. So you really should find the useful thing and then concentrate on that. Then we would try to make the point that okay - in a way such data collection is not enough but you really need to find the correct problem that you actually need to solve. [DS3]

Limited interaction with domain experts: In most cases, the domain experts would be the ones to evaluate and sometimes use the data science results. When defining the problem, the participants expressed that it was important to have input from the customers' domain experts. The domain experts know the problem best and are able to describe it very well-but their input was not readily available.

We might have a communication problem with the customer since we're not experts on the domain. We don't know what their problems are. And on the other hand they might not be aware of what we could do. [DS1]

The other one [i.e., problem] is how much we can actually communicate with the domain expert. [DS2] 
Preference for tools as a solution: The participants found that there was a general bias towards tools and products in the market. Tools were seen as easy solutions to the problems as they were easy to acquire, were well-defined, easy to start using, and were perceived with less uncertainty. This hindered the customers' attitudes towards more thorough problem solving that data science requires.

I think many times the products are preferred to in a way because if you don't know the field then you actually think [of a product]. Because it's a product you can teach anybody to use it. But that's not really the case because if you don't know what you are doing or you don't know what the problem you are solving is, you put rubbish [in] and get rubbish out. I think it goes for why [the] typical thinking [is] okay, we buy a tool and then everybody can use it. [DS3]

Data collection and preparation The challenges encountered during this activity are as follows.

Limited access to the data collection process: The participants were uncomfortable with being seen as magicians that could unravel wonderful discoveries from any sort of data without knowing its context. Not only did the participants want to have access to the data, but they also felt that understanding the process through which the data was collected would be useful in evaluating the problem and achieving the desired results.

...data is produced by some process. And, what we really need to do is understand the process or, preferably intervene with the process so that we get measurements that we really are after. Not so that there's some shadow on the wall [and] we try to deduce from that - we want to set up the whole thing. [DS4]

Poor data quality: There were several factors that compromised the data quality, such as the data being random and subpar, incorrect formatting and missing attributes, values and information. One participant gave an example:

But just as a practical example, it was not a data science project per se but in one project they had this legacy database of users where they only had one field for name. And then you had one to three first names and then several different variations of surnames and then we spent two weeks to build the engine that parsed the names to extract a surname. And even after two weeks, we got like two per cent of errors. [Research manager]

The way the data was gathered might also have had a negative effect, especially if it was collected without knowledge or intention of its use in the future.

...the data is originally not for the use that we [intend] but it has been collected for other purposes, maybe as log [data] and it's a side product of a process, and it's supposed to be somehow, [a] gold mine of insights. Or useful for some specific purpose. [DS2]

The data is often scattered around the organizations, the quality is poor. [DS1]

During the feedback session, the participants said that the data quality problem was improving. This was mainly because the market was becoming more informed about data science, hence investing effort and resources to collect meaningful data that could be utilised in the future and for different purposes. 
Lack of cooperation from all required parties: We observed that some customer organizations had internal issues that hindered the participants' involvement in the projects. The issues mainly stemmed from the lack of a shared vision for the data science project amongst different departments in the customer organizations. This made it especially difficult to gather or have access to the required data.

One thing is that often the processes are lateral in the organization so that they [spread across] different branches of the organization. So there's IT and marketing and someone else involved and it's often hard to get [them] working [together]. [DS4]

Modelling There were a couple of challenges at this stage.

Lack of the required computational resources: During the focus group interview, the participants mentioned having difficulties with getting access to the IT resources and computational environments that they needed for modelling the results, particularly if the data could not be moved from the company premises.

More than so, it's difficult to get the IT resources, both the data and the computational environment that we need. Often it's difficult to get either of them or at least one of them. [DS1]

During the feedback session, the participants pointed out that the situation had improved due to cloud solutions becoming readily acceptable and accessible.

Differences in modelling and production technologies: Sometimes, there was a difference between the modelling technology and the one in which the results are applied. This led to difficulties with integrating the results in the customer's environment and required more time, effort and money. In the end, this would limit the impact of the results.

Evaluation and validation The main challenge here was an apparent gap between the data scientists and the end users of the results. The people who ordered the project and thus got the results, e.g., the business experts, were not necessarily the actual end users acting on or using the results.

Lack of feedback from the end user: There is a difference between the feedback received from the business or domain experts working in the customer company, and the real end users of the results. If the real end users are not connected to the data scientists, it makes hard for the data scientists to actually assess the progress of their results.

This is actually the number one [problem], [lack of] tight collaboration with the end result user. [DS1]

Deployment and utilization of results The data scientists were sometimes frustrated by how the customers handled the project outcomes. Sometimes, the results were not put into use which meant that the participants would never know the real impact of the results. 
The results are not utilised: Sometimes, the results were not applied. This was due to factors, such as (1) lack of cooperation between different departments, e.g., marketing and IT, (2) the business stakeholders failed to facilitate the utilization of the results if they did not understand, were not fully convinced or they did not feel confident about the results.

...I think most of the failures that we /have/ had are because the results are just never [used]. They are ready and nobody ever uses them for anything...like I said, most of the time the problem is really to get the results into use. [DS1]

What is the impact of the results? As a result of the outcomes not being utilised, the participants found it difficult to know, measure or observe the effectiveness of the results.

For the results to be useful, they [i.e., customers] have to accept that-wellthings are how they are, not how people thought they would like them to be. [DS4]

On the other hand, the participant quoted above [DS4] pointed out the fact that in order to effectively measure the impact, one would require an experimental setup which is usually 'expensive and technically heavy' to put in place. This means some considerations have to made with respect to investments towards experimentation.

Summary of the challenges The challenges we have presented above reflect the complications of applying data science in software and service engineering as experienced by the study participants. We classified the challenges into three groups, i.e., difficult, moderate, and mild problems. The groups were according to the perceived ability to solve them, as observed during the analysis. Table 4 summarises the challenges.

Table 4. Summary of the challenges

\begin{tabular}{|l|l|}
\hline Problem Group & Challenges \\
\hline Difficult & $\begin{array}{l}\text { Communicating uncertainty, identifying the right problem, lack of } \\
\text { cooperation from all required parties, lack of feedback from the end } \\
\text { user, the results are not utilised, what is the impact of the results? }\end{array}$ \\
\hline Moderate & $\begin{array}{l}\text { Unrealistic customer expectations, limited interaction with domain } \\
\text { experts, preference for tools as a solution, limited access to the data } \\
\text { collection process }\end{array}$ \\
\hline Mild & $\begin{array}{l}\text { Poor data quality, lack of required computational resources, differ- } \\
\text { ences in modelling and production technologies }\end{array}$ \\
\hline
\end{tabular}

The difficult problems were those considered hard to solve. They comprised of human and organisational aspects which are always not easy to resolve. These 
problems also seemed to be more out of the participants' control, even though the participants considered them to be very important. The moderate problems were seen as somewhat solvable with some persistent intervention from the participants. The mild problems, such as those related to data quality, computational resources, and modelling issues, were seen as clear and easily solvable.

The human and organisational nature of the difficult problems is an indication of immature markets, which have spread extremely fast to many new application domains. Some of these problems can be expected to fade with time as the misconceptions about data science get clearer and data scientists become integrated as members of software and service development teams.

\section{Discussion}

The aim of this study was to gain understanding on the use of data science to support developers and development organisations to better guide their software and service related decisions. Using a qualitative case study approach, we have presented the process used by the case company to implement data science tasks within software development and described the characteristics of the applied data science process. We also highlighted the challenges involved while conducting the data science projects.

The data science process was composed of six phases: (1) Conceptualization - where the business problem is assessed and expectations for the project are evaluated; (2) Problem definition - in which the business problem is formalised into a solvable problem; (3) Data collection and preparation - where data is acquired and formulated into a computation-ready format; (4) Modelling - data is manipulated and analysed; (5) Evaluation and validation - where the results are assessed; (6) Deployment and utilization of results - outcomes are put to use and their impact is assessed. Some of the phases in this process, e.g., data collection and preparation, are similar to phases mentioned in other data science analysis processes, i.e., discover the data [9] and acquire data [8]. However, the data science process described in this paper is unique as it dedicates attention to the assessing of the business problem.

During the study, we observed changes towards two of the challenges. Problems with respect to computational and production environments improved over time. This was mainly because the cloud was gaining trust and people's concerns about security were becoming insignificant. In addition, cloud computing capabilities support scaling needs that might arise when handling large volumes of data [9].

Another change was that the quality of data seemed to be improving as well. Digitalization may be a reason for improving data collection, making it possible to automate the data collection process and minimise errors caused by human intervention. Furthermore, people were becoming more aware of the fact that the data could be used meaningfully in the future, hence focusing on collecting data that may be of value if needed. 
One of the issues in the problem definition phase was the bias towards tools and products to solve problems. It is important to educate the markets that tools may not provide the required solution and are only useful when they are used appropriately for well known and defined problems [8]. Hence, priority should be given to allowing the data scientists to critically evaluate the problem at hand. As Bird et al. [3] state, "For new problems, deploy the data scientists before deploying tools or hardware".

Data science projects are risky because of the unknown nature of the end results. Missing data further increases this risk but the markets are now trying to collect data that has the potential to bring as much value as possible [8]. The data scientists considered the agile methods as a good means for approaching the problem iteratively together with the customer. This allows the opportunity to find out whether the problem is practical and has an actionable solution or not.

Our findings have implications for research and practice. Researchers can apply empirical methods to solve software problems using data science. Both data science and software engineering research employ varying degrees of rigour that can be combined towards more fruitful eradication of the different challenges. For example, the challenges reported in the conceptualisation and problem definition phases of the data science process could be tackled by investigating and modelling the factors influencing the customer attitudes towards data science solutions in the software industry.

Due to the increase in data across software systems, data-driven decision making is becoming more important. Data science provides many problem solving concepts, tools and techniques [2] that can help in improving different development practices in the software intensive industry.

Threats to validity. As this study is a case study and descriptive in nature, there is little evidence to support any causal relationships, thus the internal validity is not the main concern of this study. However, the results do include knowledge constructs that could be interpreted having some causal characteristics, such as the claims from the informants that iterative approach to design science process would help to overcome certain challenges. These are clearly the views of the informants and thus taken with appropriate caution if interpreted as guidelines to follow. On the other hand, however, the informants were data science experts, who have encountered the challenges in their work and thought for the possible solutions beyond the interview sessions of this study, so their claims may be more valid and justified than random opinions.

In terms of construct validity, the richness of the data from multiple interviewees and member checking the results with the informants significantly reduce the risk that major issues would have been misunderstood by the researchers. However, one issue on construct validity may rise from the varied definitions or understandings of the term data science, particularly as its interpretation beyond this study may differ from the semantics captured between the informants and the researchers, which is broader than, e.g., data collection and analytics 
only (see Figure 1). To build a basis for the credibility [13], the interviews were audio recorded, transcribed and analysed using Atlas.ti as the tool.

Our study is conducted with the case company only, although through their customer projects, the results cover data science challenges beyond the case company only. The external validity or transferability of the results beyond the case would be based on the assumption that the informants would have encountered challenges that are not particular or stemming from the context of the case company only. That is, it is very much possible that the challenges identified have relevance beyond the case as well as the ideas proposed by the informants for alleviating the challenges. However, it is clear that the potential application of the results in other cases essentially expects a knowledgeable person or persons with good expertise in their own domain in order to interpret and apply the results in their context.

\section{Conclusions}

This study contributes to the growing interest in data science across different disciplines, specifically service and software engineering. It helps both researchers and practitioners to understand the applicability of data science in service and software development and be informed about some of the impending challenges.

The difficult problems identified comprised of human and organisational aspects, whereas the moderate problems were easier to solve. The mild problems, on the other hand, e.g., poor data quality and modelling issues, were not seen as primary concerns for the data science process.

Our results also indicate that it is possible to put in place an agile and lightweight data science process that supports continuous learning while focusing on a real business problem to be solved. The experienced data scientists highlighted that it is not enough to focus on data collection and modelling. Instead, you really need to find the correct problem that you actually need to solve.

Our future work will focus on the factors influencing the successful application of data science in service and software development projects. Other researchers can perform additional empirical studies addressing the mentioned and other rising challenges as well as investigations on improving data science processes for service and software analytics. Practitioners can use our findings to support data science initiatives and activities in the companies.

\section{Acknowledgments}

This work was supported by TEKES as part of the N4S Program of DIMECC (Digital, Internet, Materials \& Engineering Co-Creation). We would also like to thank the case company Reaktor for the possibility to conduct this research. 


\section{References}

1. A. Begel and T. Zimmermann, Analyze This! 145 Questions for Data Scientists in Software Engineering, International Conference on Software Engineering, 2014, pp. $12-22$.

2. A. Bener, A.T. Misirli, B. Caglayan, E. Kocaguneli, G. Calikli, Lessons Learned from Software Analytics in Practice, book chapter appearing in The Art and Science of Analyzing Software Data, 2015, pp. 453-489.

3. C. Bird, T. Menzies and T. Zimmermann, Past, Present, and Future of Analyzing Software Data, book chapter appearing in The Art and Science of Analyzing Software Data, 1st Edition, 2015, pp. 1-13.

4. L. Cao, Data Science: A Comprehensive Overview, Submitted to ACM Computing Surveys for Review, 2016, pp. 1-42.

5. J. W. Creswell, Research Design-Qualitative, Quantitative, and Mixed-Methods Approaches (4th ed.), SAGE, 2014.

6. D. Cruzes and T. Dyba, Recommended Steps for Thematic synthesis in Software Engineering, International Symposium on Empirical Software Engineering and Measurement (ESEM), 2011, pp. 275--284.

7. T. H. Davenport and D.J. Patil, Data Scientist: The Sexiest Job of the 21st Century, Harvard Business Review, 2012, pp. 70-76.

8. D. Fisher, R. DeLine, M. Czerwinski, S. Drucker, Interactions with Big Data Analytics, appearing in the Interactions Magazine 19 (3), 2012, pp. 50-59.

9. S. Kandel, A. Paepcke, J.M. Hellerstein and J. Heer, Enterprise Data Analysis and Visualization: An Interview Study, IEEE Transactions on Visualization and Computer Graphics 18(12), pp. 2917-2926.

10. M. Kim, T. Zimmmermann, R. DeLine, and A. Begel, The Emerging Role of Data Scientists on Software Development Teams, IEEE/ACM 38th IEEE International Conference on Software Engineering, 2016, pp. 96-107.

11. J. Kontio, L. Lehtola, and J. Bragge, Using the Focus Group Method in Software Engineering: Obtaining Practitioner and User Experiences, The International Symposium on Empirical Software Engineering (ISESE), 2004, pp. 271-280.

12. P. Liamputtong, Focus Group Methodology - Principles and Practices, SAGE, 2011.

13. M. Q. Patton, Qualitative Research \& Evaluation Methods (3rd ed.), SAGE, 2002.

14. G. Strawn, Data Scientist, Computer.org, IT Pro, pp. 55-57. 